Analysis of RFA naïve patients (table 1).

\begin{tabular}{llll} 
Abstract PTU-052 Table 1 & & \\
\hline & $\begin{array}{l}\text { "true" LGD } \\
(\mathrm{n}=34)\end{array}$ & $\begin{array}{l}\text { IND ( } \mathrm{n}=13) \\
\text { (Downstaged } \\
\text { cohort) }\end{array}$ & $\begin{array}{l}\text { NDBO ( } \mathrm{n}=21) \\
\text { (Downstaged } \\
\text { cohort) }\end{array}$ \\
\hline Mean age, years & 66 & 71 & 69 \\
Male sex & $30 / 34$ & $12 / 13$ & $16 / 21$ \\
& $(88 \%)$ & $(92 \%)$ & $(76 \%)$ \\
Median progression time to HGD/ & $10($ IQR,4-16) & $19($ IQR, 11-28) & $42($ IQR 28-72) \\
IMC (months) & & & \\
Median months of follow-Up & $17($ IQR, 6-39) & $31($ IQR,15-46) & $17($ IQR,6-31) \\
\hline
\end{tabular}

Conclusion Our data shows the variability in the diagnosis of LGD from referring centres, with $32 \%$ of referrals with confirmed LGD. The cumulative incidence of progression to HGD/IMC and time to progression varied across subgroups. Confirmed LGD had a shorter progression time compared to the down-staged group (NDBO/IND). It is important to differentiate these subgroups so that decisions on surveillance/endotherapy can be more personalised and resources utilised more wisely.

\section{PTU-053 DYSPLASIA DIAGNOSIS AT BARRETT'S SURVEILLANCE - SEATTLE PROTOCOL DOMINANT STRATEGY IN REAL WORLD NON-EXPERT CENTRES}

Georgina Keyte*, Muhammad Iqbak, YKS Viswanath, Anjan Dhar. County Durham and Darlington Nhs Foundation Trust and Durham University, Darlington, UK

\subsection{6/gutjnl-2019-BSGAbstracts.266}

Introduction Detection of dysplasia at Barrett's surveillance depends on a number of factors - the endoscopist's skill, use of advanced imaging, adherence to Seattle protocol, and high risk patient factors like male sex, smoking, length of Barrett's segment and family history of oesophageal adenocarcinoma. Whether the high rates of dysplasia diagnosis reported from expert tertiary centres can be replicated in the real world is debatable.

Aim The aim of this study was to analyse the routes to dysplasia diagnosis in a dedicated Barrett's surveillance programme in County Durham, specifically looking at the grade of the endoscopist, use of advanced imaging (NBI, Acetic Acid), diagnosis by targetted biopsy vs. Seattle Protocol, and the nature of dysplasia (Low grade vs. high grade).

Methods An electronic database search of over 600 Barrett's surveillance histology was carried out for the period 20152018, extracting all reported dysplasia. The endoscopy database was then interrogated for the following: patient demographics, grade of endoscopist (medical consultant gastroenterologist, non-medical endoscopist and surgical consultant), sedated vs. unsedated procedure, visible dysplasia, HD-white light vs. image enhancement, length of Barrett's segment and Prague classification, Paris classification of any visible lesions, targeted vs. Seattle Protocol biopsies, and grade of dysplasia. All endoscopies were carried out using high-resolution scopes and where a visible lesion was identified a targeted biopsy was taken.

Results 94 patients with dysplasia were analysed, M:F ratio 3.5:1, mean age 71 yrs. Barrett's length ranged from 1-14 $\mathrm{cm}$, with 32\% endoscopists reporting the Prague classification. Surveillance was done by: consultant gastroenterologists (48pts), nurse endoscopists (42pts) and consultant surgeons (4pts). Although only $3.2 \%$ endoscopists explicitly mentioned a Seattle biopsy protocol in their report, histology showed that protocol had been followed in 51\% of endoscopies. $52 \%$ consultant gastroenterologists, $45 \%$ nurse endoscopists and $100 \%$ consultant surgeons adhered to Seattle biopsy protocol. The distribution of low grade dysplasia (LGD):high grade dysplasia (HGD):carcinoma- in-situ (situ) in the Seattle group 21:21:6 compared with a random biopsy protocol of 8:19:19. Image enhancing techniques were used in just $4 \%$ pts.

Conclusions In this real world NHS study, we found that after 4 years of the BSG guidelines, visible dysplasia is extremely difficult to detect. The Seattle biopsy protocol was followed in only $51 \%$ of endoscopies. There is a need for quality improvement \& training for Barrett's surveillance amongst medical and non-medical endoscopists, including image enhanced surveillance and chromoendoscopy.

\section{PTU-054 IN CHILDREN WITH EOSINOPHILIC OESOPHAGITIS, SYMPTOM RESOLUTION DOES NOT CORRESPOND WITH ENDOSCOPIC OR HISTOLOGICAL IMPROVEMENT}

${ }^{2}$ Arati Rao, ${ }^{2}$ Yasir Alrusayni, ${ }^{2}$ Babu Vadamalayan, ${ }^{2}$ Dhamyanthi Thangarajah, ${ }^{2}$ Maesha Deheragoda, ${ }^{2}$ Ben Hope. ', Queen Mary University of London, London, UK; ${ }^{2}$ King's College Hospital, London, UK

\subsection{6/gutjnl-2019-BSGAbstracts.267}

Introduction Eosinophilic esophagitis (EoE) is a chronic, local immune-mediated condition characterised by symptoms relating to oesophageal dysfunction and histologically by eosinophilpredominant inflammation. In this study, we describe symptomatic, endoscopic and histological response to treatment in children attending a tertiary paediatric gastroenterology centre. Methods Retrospective analysis of all children (age $<18$ yrs) diagnosed with EoE from 1/1/12 to 6/1/17 with $\geq 1$ year follow-up. Diagnosis was based on the presence of $\geq 15$ eosinophils/high powered field (eos/hpf) in endoscopic oesophageal biopsies. Children with mucosal eosinophilia elsewhere in the gut were excluded. Histological review was carried out by an independent pathologist. Data was analysed using SPSS 21 (Armonk, New York, NY, USA) and Prism 7 (San Diego, CA, USA). Significance was two-tailed and defined as $\mathrm{p}<0.05$.

Results Of the 25 children who met the criteria, the mean age at diagnosis was 5.8 yrs with a mean symptom duration of 16.4 months, and a mean eos/hpf count of 26.3. The commonest presenting symptoms were vomiting and abdominal pain. All patients received a PPI; 22 were prescribed dietary therapy; and 16 topical steroids.

There was a high rate of symptomatic improvement, with 18 patients denying any gut symptoms at latest follow-up. However, even within this group a significant fall in eos/hpf count between diagnosis and follow up endoscopies was not observed (figure 1). This was seen regardless of the treatment modality used: dietary manipulation before and after mean [SD] 26.9 [15.1] vs 23.9 [20.0], $\mathrm{p}=0.4$; topical steroids mean [SD] 31.4 [14.1] vs 28 [19.1], $\mathrm{p}=0.5$; both concurrently mean [SD] 32.8 [14.4] vs 30.2 [19.7], $\mathrm{p}=0.7$. Overall, $17 / 25(68 \%)$ still had an eos/hpf count of $>15$. Only $20 \%$ had macroscopically and histologically normal mucosa at follow up endoscopy. None of the children had a stricture at diagnosis or developed one during follow up. 


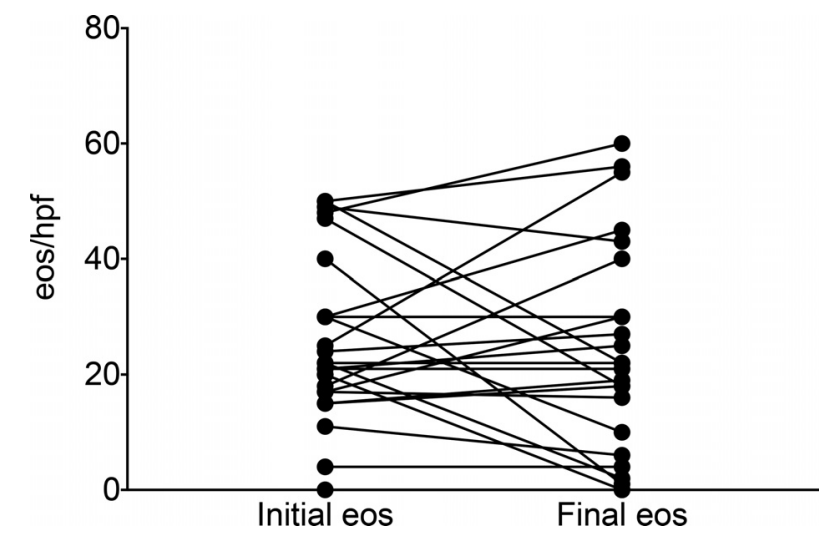

Abstract PTU-054 Figure 1 EOS Baseline and end of follow-up period

Conclusions Despite a high rate of symptom improvement, in only a small minority did the eos/hpf count fall below the diagnostic level. Normalisation of endoscopic appearance was also less common that has been reported.

It has been proposed that persistent eosinophilia leads to fibrotic tissue remodelling and disease progression. Longer term studies with larger cohorts are needed to confirm this hypothesis. Until then it will remain unclear whether more aggressive intervention is indicated in asymptomatic patients.

\section{PTU-055 COMPARATIVE COST-EFFECTIVENESS OF GASTROESOPHAGEAL REFLUX DISEASE (GORD) CENTRIC AND POPULATION BASED BARRETT'S OESOPHAGUS (BO) SCREENING}

\footnotetext{
1,2Sarmed Sami*, ${ }^{2} J a m e s$ Moriarty, ${ }^{2} J o r d a n$ Rosedahl, ${ }^{2}$ Bijan Borah, ${ }^{2} J o h n$ Kisiel, ${ }^{2}$ David Katzka, ${ }^{2}$ Kenneth Wang, ${ }^{2}$ David Ahlquist, ${ }^{2}$ Prasad Iyer. ${ }^{1}$ University College London, London, UK; ${ }^{2}$ Mayo Clinic, Rochester, USA
}

\subsection{6/gutjnl-2019-BSGAbstracts.268}

Introduction With the development of minimally invasive tests for $\mathrm{BO}$, the prospect of population screening (all adults age $\geq 50$ ) instead of targeted screening (white, male, age $\geq 50$, GORD) has arisen. We aimed to evaluate the comparative cost effectiveness of current tests utilising recently published data on prevalence, accuracy, screening uptake, and costs.

Methods Decision analytic methods were conducted using Markov Modeling. The simulation compared six screening strategies to no screening: sedated OGD (sOGD, Cytosponge with TFF3 biomarker, Sponge on String (SoS) with methylated DNA markers, breath testing (E-Nose), hospital-based transnasal endoscopy (hTNE), and mobile unit-based transnasal endoscopy (mTNE). The model simulated a hypothetical cohort of 100,000 individuals aged $\geq 50$ followed up for 40 years. sOGD was used as a confirmatory test of positive findings for all other diagnostic tests. Mean costs (direct as well as indirect costs resulting from missed or false positive diagnoses and low test uptake) were calculated for each strategy. Quality of life outcomes were based on quality adjusted life years (QALYs).

Results Screening (GORD centric and population) was associated with small gains in QALYs compared to no screening (table 1). Under the general population analysis, mean costs ranged from $\$ 120$ (mTNE) to $\$ 554$ (E-Nose). While mTNE was the dominant strategy, both the Cytosponge and SoS tests had the lowest incremental cost effectiveness ratios (ICERs) (table 1). Under the GORD centric screening analysis, mean costs ranged from $\$ 212$ (mTNE) to $\$ 768$ (E-Nose). Cytosponge, SoS, hTNE, and mTNE were all dominant strategies compared to no screening. Out of those, mTNE was the least costly and most effective strategy. The E Nose test had the highest ICER primarily due to lower accuracy estimates.

Conclusions While mTNE was the dominant strategy for both screening scenarios, this technique is not widely utilised and has been only evaluated in one centre. The Cytosponge and SoS strategies had very favourable ICERs in both strategies and may make population based $\mathrm{BO}$ screening regardless of GORD status feasible.

\begin{tabular}{|c|c|c|c|}
\hline \multicolumn{4}{|c|}{ Population screening (adults, age $\geq 50$ ) } \\
\hline & Cost $\$$ & QALY & ICER \$ \\
\hline sOGD & 413 & 39.6227 & 52,000 \\
\hline Cytosponge & 166 & 39.6216 & 3,333 \\
\hline Sos & 175 & 39.6221 & 5,000 \\
\hline hTNE & 218 & 39.6207 & 21,667 \\
\hline mTNE & 120 & 39.6207 & Dominant \\
\hline E-Nose & 554 & 39.6226 & 81,837 \\
\hline No screening & 153 & 39.6177 & NA \\
\hline \multicolumn{4}{|c|}{ GORD centric screening (white men $\geq 50$ with chronic reflux) } \\
\hline & Cost \$ & QALY & ICER \$ \\
\hline sOGD & 486 & 39.336 & 26,078 \\
\hline Cytosponge & 280 & 39.3365 & Dominant \\
\hline SoS & 313 & 39.3379 & Dominant \\
\hline hTNE & 310 & 39.338 & Dominant \\
\hline mTNE & 212 & 39.338 & Dominant \\
\hline E-Nose & 768 & 39.3325 & 259,375 \\
\hline No screening & 353 & 39.3309 & NA \\
\hline
\end{tabular}

\section{PTU-056 GENERALIST VS SPECIALIST: DOES DEDICATED ENDOSCOPY IN BARRETT'S OESOPHAGUS YIELD IMPROVED ADHERENCE TO BSG GUIDANCE?}

Samuel Stroud*, Kumar Basu. Sheffield Teaching Hospitals NHS Trust, UK

\subsection{6/gutjnl-2019-BSGAbstracts.269}

Introduction Barrett's oesophagus (BO) is a premalignant precursor of oesophageal adenocarcinoma (OAC). ${ }^{1}$ With yearly progression seen in 0.3-0.6/100 patients, a robust surveillance strategy is crucial. ${ }^{1}$ The British Society of Gastroenterology (BSG) 2013 guidance recommends the use of two techniques for reviewing non-dysplastic BO: (1) Prague Classification and (2) Seattle Biopsy Protocol. $^{2}$ A retrospective analysis (20142015) at a large teaching hospital showed correct use of 'Prague Classification' and 'Seattle Biopsy Protocol' to be $32 \%$ and 42\%, respectively. Subsequently, recommendations to introduce specialist (operators with BO interest who are highly familiar with $B S G$ guidance) endoscopy as well as improving the general familiarity with the BSG guidance were proposed. This study aimed to evaluate (1) overall compliance to guidance for both techniques, (2) differences between specialist and generalist operators, and (3) availability of specialist endoscopy. 